\title{
The effects of flavanone-rich citrus juice on cognitive function and cerebral blood flow: an acute, randomised, placebo-controlled cross-over trial in healthy, young adults
}

\author{
Daniel J. Lamport ${ }^{1}$, Deepa Pal ${ }^{1}$, Anna L. Macready ${ }^{2}$, Sofia Barbosa-Boucas ${ }^{1}$, John M. Fletcher ${ }^{3}$, \\ Claire M. Williams ${ }^{1}$, Jeremy P. E. Spencer ${ }^{2}$ and Laurie T. Butler ${ }^{1 *}$ \\ ${ }^{1}$ School of Psychology and Clinical Language Sciences, University of Reading, Reading RG6 6AL, UK \\ ${ }^{2}$ Molecular Nutrition Group, School of Chemistry, Food and Pharmacy, University of Reading, Reading RG6 6AP, UK \\ ${ }^{3}$ PepsiCo, 100 Summit Lake Drive, Valhalla, NY 10595, USA
}

(Submitted 9 May 2016 - Final revision received 14 November 2016 - Accepted 16 November 2016)

\section{Abstract}

A plausible mechanism underlying flavonoid-associated cognitive effects is increased cerebral blood flow (CBF). However, behavioural and $\mathrm{CBF}$ effects following flavanone-rich juice consumption have not been explored. The aim of this study was to investigate whether consumption of flavanone-rich juice is associated with acute cognitive benefits and increased regional CBF in healthy, young adults. An acute, single-blind, randomised, cross-over design was applied with two 500-ml drink conditions - high-flavanone (HF; 70.5 mg) drink and an energy-, and vitamin C- matched, zero-flavanone control. A total of twenty-four healthy young adults aged 18-30 years underwent cognitive testing at baseline and 2-h after drink consumption. A further sixteen, healthy, young adults were recruited for functional MRI assessment, whereby $\mathrm{CBF}$ was measured with arterial spin labelling during conscious resting state at baseline as well as 2 and $5 \mathrm{~h}$ after drink consumption. The HF drink was associated with significantly increased regional perfusion in the inferior and middle right frontal gyrus at $2 \mathrm{~h}$ relative to baseline and the control drink. In addition, the HF drink was associated with significantly improved performance on the Digit Symbol Substitution Test at $2 \mathrm{~h}$ relative to baseline and the control drink, but no effects were observed on any other behavioural cognitive tests. These results demonstrate that consumption of flavanone-rich citrus juice in quantities commonly consumed can acutely enhance blood flow to the brain in healthy, young adults. However, further studies are required to establish a direct causal link between increased CBF and enhanced behavioural outcomes following citrus juice ingestion.

\section{Key words: Flavonoids: Juices: Cognition: Cognitive function: Cerebral blood flow: Functional MRI}

Studies investigating the neuroprotective effects of foods and beverages containing flavonoids suggest that they may lead to benefits regarding memory and learning by improving neuronal functioning and promoting neuronal protection and regeneration ${ }^{(1)}$. In rodents, dietary flavanone supplementation (e.g. hesperidin) over several weeks is associated with significant improvements in spatial working memory. Moreover, these cognitive improvements correlate with increased expression of signalling proteins involved in learning and memory, as well as increased brain-derived neurotrophic factor (BDNF) in the hippocampus ${ }^{(2,3)}$. These are important findings, as increased expression of BDNF is associated with benefits for cognitive function in humans such as slower onset of Alzheimer's disease ${ }^{(4)}$. This supports the presence of mechanistic pathways by which citrus fruit-based flavanones may have positive effects on the brain.

Epidemiological data showing an association between flavanone consumption and crystallised intelligence ${ }^{(5)}$ are supported by positive effects from several human intervention studies indicating cognitive benefits in adults following chronic consumption of flavanone-rich fruits and vegetables ${ }^{(6,7)}$. For example, improved memory function in older adults with mild cognitive impairment (MCI) has been observed following daily consumption of concord grape juice for $12^{(8)}$ and 16 weeks ${ }^{(9)}$. Of particular relevance here is a recent finding that daily consumption of flavanone-rich orange juice for 8 weeks was associated with improvements in executive function and episodic memory in healthy, older adults aged $60-81$ years ${ }^{(10)}$. This indicates that consumption of fruit juices that contain flavanones as the predominant flavonoid may lead to benefits for the human brain, even in healthy adults.

Neuroimaging studies in young, human adults have demonstrated that consumption of flavanol-rich cocoa can acutely enhance peripheral and cerebral blood flow $(\mathrm{CBF})^{(11,12)}$. Furthermore, promising associations have been observed

Abbreviations: CBF, cerebral blood flow; CT, control; DSST, Digit Symbol Substitution Test; fMRI, functional MRI; HF, high flavanone.

* Corresponding author: Professor L. T. Butler, email 1.t.butler@reading.ac.uk 
between increased neuronal activity and behavioural benefits following chronic flavanol-rich cocoa supplementation. Enhanced activation in the dentate gyrus (measured with a functional MRI (fMRI) blood oxygenation level-dependent (BOLD) signal) and simultaneous improvements in spatial working memory were reported in healthy, older adults following consumption of flavanol-rich cocoa for 3 months relative to a low-flavanol control $^{(13)}$.

However, other chronic flavanol interventions have failed to report concomitant cognitive benefits in the presence of enhanced neuronal activation. For example, increased steady state-evoked potentials (assessed using Steady-State Probe Topography) in the posterior parietal and central-frontal regions were observed in middle-aged adults following $30 \mathrm{~d}$ daily consumption of 250 - or $500-\mathrm{mg}$ cocoa flavanol drinks relative to placebo; however, there were no effects for behavioural measures of spatial working memory ${ }^{(14)}$. Similarly, enhanced activation was observed in various brain regions during performance of an attention-switching task following five days' consumption of 172-mg cocoa flavanols. However, changes in the BOLD signal were not associated with performance on the attention-switching task ${ }^{(12)}$.

To summarise, the evidence suggests that flavonoid consumption can enhance vasodilation in the periphery and lead to increased blood flow in specific regions of the brain in the acute postprandial period. Daily flavonoid consumption over several weeks is associated with cognitive benefits, but as yet there is only weak evidence supporting a coupling between increased $\mathrm{CBF}$ and improved performance on neuropsychological tests. The present study builds upon these findings by investigating whether the aforementioned positive cognitive effects of daily flavanone consumption over several weeks ${ }^{(10)}$ are supported by acute cognitive benefits in the immediate postprandial phase. It is reasonable to hypothesise that acute cognitive benefits are underpinned by changes in CBF. Therefore, in addition to assessing behavioural outcomes, the present study examined the effects of flavanone-rich juice on CBF using fMRI arterial spin labelling (ASL). We chose a commercially available citrus-based juice, given that flavanones are naturally found in high concentrations in citrus fruits such as orange and grapefruit. This also reflects the quality and quantity of juice consumed by the general population. In sum, the aim of the present study was to investigate the effects of flavanone-rich juice on acute cognitive function and CBF in healthy, young adults by adopting a placebo-matched, cross-over, randomised, single-blind, design.

\section{Methods}

Different participants were recruited for the behavioural cognitive arm ( $n$ 28) and the ASL imaging arm ( $n$ 16) of the study (see Table 1); however, inclusion and exclusion criteria were identical for both arms. Participants were not permitted to take part in both arms. At the time of designing the study, there was absence of published data concerning the effects of flavanone consumption in humans on cognitive function, cardiovascular outcomes or CBF. Therefore, we considered it important to create an experimental design in which cognitive and CBF effects could
Table 1. Participant characteristics for the behavioural cognitive arm and the arterial spin labelling (ASL) arm

(Mean values and standard deviations)

\begin{tabular}{|c|c|c|c|c|c|}
\hline & \multicolumn{2}{|c|}{$\begin{array}{l}\text { Behavioural cognitive } \\
\text { arm ( } n \text { 24) }\end{array}$} & \multicolumn{2}{|c|}{$\begin{array}{l}\text { ASL arm } \\
(n 16)\end{array}$} & \multirow{2}{*}{$\begin{array}{l}P_{\text {comparison }} \\
\text { between arms }\end{array}$} \\
\hline & Mean & SD & Mean & $\mathrm{SD}$ & \\
\hline Age (years) & 22 & $2 \cdot 2$ & 22 & 1.9 & 0.73 \\
\hline $\mathrm{BMI}\left(\mathrm{kg} / \mathrm{m}^{2}\right)$ & $23 \cdot 2$ & 3.9 & $23 \cdot 3$ & $1 \cdot 7$ & 0.88 \\
\hline Years in education & $16 \cdot 9$ & 1.8 & $16 \cdot 6$ & 1.4 & 0.53 \\
\hline MMSE (max 30) & $29 \cdot 3$ & 1 & $29 \cdot 6$ & 0.5 & 0.19 \\
\hline
\end{tabular}

MMSE, Mini Mental State Examination.

be examined in isolation. For, example, it is important to establish whether the effects on $\mathrm{CBF}$ are observed independently of behavioural effects. Furthermore, in light of the absence of experimental support for a specific behavioural task sensitive to flavanone consumption in humans, it was considered that a range of cognitive functions should be assessed. Incorporating a comprehensive cognitive battery into the fMRI sequencing schedule posed significant practical difficulties. Therefore, a decision was taken to recruit separate cohorts for the behavioural and imaging arms. Healthy, young adults aged 18-30 years were recruited from the University of Reading and surrounding areas via community advertising with posters, leaflets and emails. A total of twenty-four participants (four males) completed the behavioural cognitive arm (four participants dropped out because of work commitments or illness), and all sixteen participants completed the ASL arm (eight males). Inclusion criteria were BMI $19-25 \mathrm{~kg} / \mathrm{m}^{2}$ and fluency in English, whereas exclusion criteria were signs of MCI (Mini Mental State Examination (MMSE) Score <26), smoking, alcohol consumption $>15$ units/week, orange juice consumption $>250 \mathrm{ml} / \mathrm{d}$, fruit/vegetable consumption $>4$ portions $/ \mathrm{d}$, caffeine intake $>3$ drinks/d, actively pursuing weight loss through a dietary intervention, clinical diagnosis of mental illness, neurological disease, chronic fatigue, kidney disease, liver disease, thyroid dysfunction, diabetes mellitus, myocardial infarction or hypertension, and consumption of medication for lipids, hypertension, hypotension or anticoagulation. Recruitment commenced from March 2011 and was terminated in August 2011. Our sample size was based on previous studies reporting significant cognitive effects of berry flavonoids in older adults with sample sizes ranging from nine to twenty-one ${ }^{(8,9,15)}$ and improvements in $\mathrm{CBF}$ following intake of cocoa flavanols in sixteen young adults ${ }^{(12)}$.

\section{Design}

An acute single-blind, randomised, cross-over design was applied with two drink conditions - high flavanone (HF) and control (CT). Cognitive behavioural testing and ASL measurements were performed before and after consumption of the drink at each visit (see procedure). The 500-ml HF drink was a commercially available $100 \%$ juice (Tropicana Ruby Breakfast Juice; PepsiCo Inc.), which naturally contained $70.5-\mathrm{mg}$ flavonoids (42.15-mg hesperidin, $17 \cdot 25-\mathrm{mg}$ naringin, $6 \cdot 75-\mathrm{mg}$ narirutin, $4 \cdot 3-\mathrm{mg}$ caffeic acid; analysed by the University of Reading), $941 \mathrm{~kJ}$ (225 kcal), 48.5-g sugars, 4-g protein, 0-g fat, 
3.5-g fibre and 150-mg vitamin C. The Tropicana Ruby Breakfast Juice contained juices from oranges and grapefruits. The 500-ml CT drink was a commercially available concentrated cordial product (Lemon Barley Squash; Sainsbury's), which was prepared with $240 \mathrm{ml}$ of concentrate and $260 \mathrm{ml}$ of mineral water (Buxton Spring still mineral water; Nestlé) containing zero flavonoids, $962 \mathrm{~kJ}$ ( $230 \mathrm{kcal})$, 48-g sugars, $0 \cdot 7-\mathrm{g}$ protein, $0-\mathrm{g}$ fat, $0 \cdot 3-\mathrm{g}$ fibre and $130 \mathrm{-mg}$ vitamin C. Our dose of $70 \cdot 5-\mathrm{mg}$ flavonoids could be considered low relative to previous studies ${ }^{(6)}$; however, it is important to examine whether cognitive benefits are associated with consuming concentrations of flavanones, which are present in the habitual diet. Therefore, the 500-ml juice serving provided an acceptable balance between a suitable flavonoid concentration and an achievable volume of consumption within the context of the habitual diet. The drinks were stored at $4^{\circ} \mathrm{C}$ and prepared and served by the experimenter. Each 500-ml portion was served in two 250-ml opaque flasks and consumed through an opaque straw, thus participants could not see the drink and remained blinded. The randomisation order was determined by an independent statistician. For the behavioural cognitive arm, twelve participants consumed the HF drink at visit 1 and twelve consumed the CT drink at visit 1, whereas for the ASL arm eight participants consumed the HF drink at visit 1 and eight consumed the CT drink at visit 1 .

\section{Procedure}

In summary, participants attended three separate visits - one screening visit and 2 test-day visits. The behavioural arm test days included two cognitive test time points (baseline and $2 \mathrm{~h}$ later), and the ASL arm visit days included three time points (baseline, $2 \mathrm{~h}$ later and $5 \mathrm{~h}$ later). The screening visit and each test-day visit were separated by a 1 -week washout period. Initially, telephone screening interviews were performed, and volunteers who met inclusion criteria were invited to attend the University of Reading Hugh Sinclair Nutrition Unit for a screening visit. At screening, data on height, weight, health status, medication and blood pressure were collected, and participants completed the MMSE, a diet and lifestyle questionnaire and a fruit and vegetable questionnaire, data from which were used to corroborate inclusion/exclusion criteria. For each test-day visit, participants arrived at 08.00 hours after having abstained from alcohol for $48 \mathrm{~h}$ and all other food and drinks (except water) for $12 \mathrm{~h}$. At screening, participants were provided with low-nitrate bottled water for consumption during the fast. Before each test-day visit, participants were also instructed to avoid polyphenol-rich foods for $24 \mathrm{~h}$ (including berries, fruits, fruit juices, jams and preserves, red wine, black, green and fruit teas, coffee, cocoa, soya products, caffeinated energy drinks and vegetables except potatoes), and were provided with standardised typed instructions identifying which foods to avoid. The evening before each test day, participants consumed (at home) a low-fat, standardised chicken and rice meal provided by the research team $(1464 \mathrm{~kJ}$ (350 kcal), 6.9-g fat of which $3 \mathrm{~g}$ of saturates, $52 \cdot 1 \mathrm{-g}$ carbohydrate of which $9 \cdot 7-\mathrm{g}$ sugars, $19 \mathrm{~g}$ of protein, $1.4 \mathrm{~g}$ of fibre, $0.9 \mathrm{~g}$ of salt) to avoid second-meal cognitive effects ${ }^{(16)}$. On each test day, participants were required to orally confirm that they had adhered to the aforementioned dietary restrictions. Following a 15-min rest period, blood pressure measurements were taken (on behavioural visit days only) on the left upper arm by a validated blood pressure monitor (Omron MX2 automatic digital upper arms BP monitor) and recorded as the average of three consecutive measurements. At 08.30 hours, participants consumed a standardised breakfast within $15 \mathrm{~min}$ (88-g croissant, 25-g cream cheese and 120-ml bottled mineral water, containing 51-g fat, 14-g protein, 64-g carbohydrates, $3251 \mathrm{~kJ}$ ( $777 \mathrm{kcal})$ ). For the behavioural test days, baseline cognitive testing commenced at 08.45 hours, followed by consumption of the drink (either HF or CT) at 09.45 hours. Participants were informed that the drink was a fruit-based beverage available in most UK supermarkets and which must be consumed within $15 \mathrm{~min}$. Blood pressure was measured at 11.40 hours (behavioural arm only), and lunch, identical to breakfast in both content and amount, was provided $15 \mathrm{~min}$ before the 2 -h post-drink cognitive battery, which commenced at 12.00 hours. An assessment at this time point was based on previous data demonstrating cognitive effects $2 \mathrm{~h}$ following an acute flavonoid dose ${ }^{(12)}$. For the ASL visit days, the timings were identical to the behavioural cognitive visit days, such that ASL measurements were performed at 08.45 hours (baseline), 12.00 hours $(2 \mathrm{~h}$ ) and 15.00 hours $(5 \mathrm{~h})$. The behavioural cognitive visits took place in individual cubicles at the University of Reading Hugh Sinclair Nutrition Unit, and the ASL visits took place at the Centre for Integrative Neuroscience and Neurodynamics (CINN). Participants remained within the Nutrition Unit or the CINN for the entire test visit during which only water consumption was permitted (notwithstanding the test-day foods and drinks). Participants received a $\$ 120$ honorarium upon completion. This study was conducted according to the guidelines laid down in the Declaration of Helsinki, and all procedures involving human subjects were approved by the School of Psychology and Clinical Languages Ethics Committee. Written and verbal informed consent were obtained and formally recorded.

\section{Cognitive battery}

The 45-min cognitive battery consisted of the following tests administered in the respective order: Freiburg Vision Test (version 3.6.3), Word Recall (immediate), Logical Memory (immediate recall), Sequence Learning Task, Digit Symbol Substitution Test (DSST), Stroop Test, Letter Memory Test, Go-NoGo Task, Spatial Delayed Recall, Word Recall (delayed) and Logical Memory (delayed). Where multiple versions of a test were required (see below), parallel versions were presented in a counter-balanced order across conditions and visits. The Freiburg Vision Test assesses visual acuity ${ }^{(17)}$ for which there are two dependent variables - Landolt $\mathrm{C}$ and Vernier Threshold. To acquire the Landhold C measurement, participants were required to identify the orientation of a horseshoe symbol using the numbers 1-9 on the keyboard keypad (excluding 5). The presentation size of the horseshoe, and thus the ease of identifying the orientation, randomly varied across trials. Landolt $\mathrm{C}$ was subsequently calculated according to the number of correct responses relative to the presentation size. To acquire the Vernier Threshold, participants viewed a 
stimulus that consisted of two $1-\mathrm{cm}$ lines with one directly above the other. Participants pressed the left scroll key if the line above was to the left of the line below, and the right scroll key if the line above was to the right of the line below. The degree to which the lines were aligned varied randomly across trials. The Vernier Threshold was subsequently calculated according to the number of correct responses relative to the horizontal distance between the two lines ${ }^{(17)}$. Verbal Recall involved computerised, individual presentation of thirty words. A response was required (using the keys ' $M$ ' for yes, ' $\mathrm{Z}$ ' for no) according to one of five questions that required visual, phonetic or semantic processing of the target word (e.g. 'is the word in capitals', 'does the word rhyme with ...' or 'is the word a type of ...'). Upon cessation of the presentation, oral recall of the target words was required (the dependent variable). Within each version of the test, each word was accompanied by the same question for all participants, whereas the order of presentation varied randomly. Equal versions were created and matched for frequency, familiarity, imageability, meaningfulness, word length and syllables. Delayed Word Recall involved one attempt to orally recall the words presented $30 \mathrm{~min}$ earlier during Immediate Word Recall. The Logical Memory Test (Wechsler Memory Scale - Revised) requires oral recall of a short paragraph. The paragraphs were presented via cassette tape. The dependent variables for immediate and delayed recall were the number of correctly recalled units. The Sequence Learning Task ${ }^{(18)}$ required participants to immediately press the keys ' $\mathrm{V}, \mathrm{B}, \mathrm{N}$ or $\mathrm{M}$ ' according to the appearance of a stimulus (a 2-mm white dot for $200 \mathrm{~ms}$ ) in one of four $3.5 \times 2-\mathrm{cm}$ boxes on the screen. Unbeknownst to participants, the order of stimulus presentation followed a set sequence (one block); thus, this test assesses the ability to learn a sequence. The duration of each repetitive sequence varied from two to four trials. Each test presentation contained six blocks, with each block consisting of 100 trials. The dependent variable was number of correct responses. The $\operatorname{DSST}^{(19)}$ is a pen and paper test that contains a key of nine digit-symbol pairs and an accompanying list of digits. Under each listed digit, a space is provided to enter the corresponding symbol. Participants entered as many symbols as possible over $90 \mathrm{~s}$. The dependent variable was the number of correct responses. The computerised Stroop Test ${ }^{(20)}$ required participants to identify the colour in which a word was presented. There were 120 randomly presented stimuli, each for $1650 \mathrm{~ms}$, consisting of sixty congruent and sixty incongruent trials (a congruent trial being when the meaning of the word matched the colour in which it was presented). Participants responded with the keys 1-4, which represented the colours green, blue, red and yellow, respectively. The dependent variable was reaction time (for correct responses only). The Letter Memory Task ${ }^{(21)}$ involved serial 2000-ms presentation of individual letters. The number of letters per trial varied randomly between 5, 7, 9 and 11 for a total of twelve trials and forty-eight letters. For each trial, at the termination of the presentation phase, participants were required to orally recall the final four letters from the presentation. The dependent variable was the total number of correct responses defined as recalling the correct sequence in its entirety. The Go-NoGo is a computerised task assessing inhibition and sustained attention. The present version was adapted from the Go-NoGo paradigm ${ }^{(22)}$. Participants were required to respond to sixty stimuli using one of three specified keyboard keys - 'p' 'q' or 'space bar'. The stimuli consisted of X, Y or a number 'lure'. Initially, there was a 25-stimuli 'Pre-Potent Go' phase. During the Pre-Potent Go phase, $\mathrm{X}$ and $\mathrm{Y}$ were presented alternately, with the participant required to press ' $\mathrm{q}$ ' when $\mathrm{X}$ appeared and ' $\mathrm{p}$ ' when $\mathrm{Y}$ appeared. The $\mathrm{X}$ and $\mathrm{Y}$ were known as the 'Go' trials. The Go-NoGo phase followed the Pre-Potent Go phase. During the Go-NoGo phase, the 'Go' trials were interspersed with 'NoGo' trials; these appeared as number lures. Pressing the space bar was the required response upon viewing a number lure. During the Go-NoGo phase, $\mathrm{X}$ and $\mathrm{Y}$ were presented randomly, interspersed with number lures, such that the predictable alternating sequence was disrupted. Responses were required only if a Y appeared after an X or viceversa, and therefore the participant must inhibit the established pre-potent response in all other trials. Reaction Time for correct responses was the dependent variable. The Spatial Delayed Recall Test required participants to recall the location of a white dot on the screen. Each trial commenced with a fixation cross followed by presentation of a white dot for $50 \mathrm{~ms}$ in a random location. The white dot was replaced by a randomly generated number between 90 and 99 at which point participants were asked to orally subtract three from this number continuously for $8 \mathrm{~s}$. Once $8 \mathrm{~s}$ had elapsed, the number disappeared and the participant was required to indicate (by touching the screen) the location at which the white dot had previously appeared. There were sixteen trials in total, and the dependent variable was the distance from the target $(\mathrm{mm})$.

\section{Functional MRI protocol}

Scanning was performed at the CINN, University of Reading, using a 3.0-T Siemens MAGNETOM Trio MRI scanner with a 12-channel Head Matrix coil. The ASL images were acquired using the PICOREQ2T sequence with the following parameters: number of slices $=18$, slice thickness $=5.0 \mathrm{~mm}$, inter-slice gap $=1.25 \mathrm{~mm}$, repetition time $(\mathrm{TR})=2500 \mathrm{~ms}$, echo time $(\mathrm{TE})=11 \mathrm{~ms}$, inversion time (TI) $1=700$, saturation stop time $=1600$, TI $2=1800$ and perfusion mode $=$ PICOREQ2T (pulsed). A high-resolution whole-brain three-dimensional anatomical image was also acquired using a magnetisation-prepared rapid gradient echo (MPRAGE) gradient sequence, with $176 \times$ 1-mm-thick slices $(1 \times 1 \times 1$ voxels size, TE: $2.52 \mathrm{~ms}$, TR: $2020 \mathrm{~ms}$, TI: $1100 \mathrm{~ms}$, field of view: $250 \times 250$, slice thickness: $\mathrm{mm}^{2}$, flip angle: $9^{\circ}$ ). fMRI data processing was carried out using FMRI Expert Analysis Tool (FEAT) version 5.98, part of FSL (FMRIB's Software Library; www.fmrib.ox.ac.uk/fsl). ASL volumes from each scanning session were all registered to the corresponding individual's high-resolution structural image using rigid body transformations. In a second step, the images were registered to the Montreal Neurological Institute (MNI) template brain using a $12 \mathrm{df}$ affine transformation algorithm. To allow voxel-wise comparisons, each CBF map was individually processed using perfusion signal modelling, which models the differences between control images and tagged (spin labelled) images within a time series. A CBF map was produced for each participant, drink (HF and CT) and time point (baseline, $2 \mathrm{~h}$ and $5 \mathrm{~h}$ ). 


\section{Statistical analysis}

All analyses and data processing were performed by independent researchers who did not participate in any of the test-day procedures and remained blinded to the condition. Cognitive test and blood pressure-dependent variables were assessed using a $2 \times 2$ repeated-measures ANOVA (drink $\times$ time). Significant main effects and interactions were explored with post hoc $t$ tests applying Bonferroni corrections for family-wise error. Analyses of the cognitive and blood pressure data were performed using SPSS Statistics 21. fMRI data processing was carried out using FEAT version 5.98, part of FSL (FMRIB's Software Library, www.fmrib.ox.ac.uk/fsl). ASL volumes from each scanning session were all registered to the corresponding individual's high-resolution structural image using rigid body transformations. In a second step, the images were normalised to the MNI template brain using a 12 degrees of freedom affine transformation algorithm. To allow voxel-wise comparisons, we first processed each CBF map individually using the perfusion signal modelling, which models the differences between control and tag. We processed a CBF map for each participant, time point (pre and post) and drink (HF and CT). These perfusion flow maps were then given as inputs for the second-level analysis ( $t$ contrasts), which processed the difference before and after consumption of each drink. Specifically, these $t$ test contrasts compared the CBF maps at 2 and $5 \mathrm{~h}$ after drinks with the pre-drink baseline, and had the form of a simple subtraction defined as $\mathrm{CBF} 2 \mathrm{~h}-\mathrm{CBF}$ baseline and $\mathrm{CBF} 5 \mathrm{~h}-\mathrm{CBF}$ baseline. The output of this second step was contrast images, which corresponded to the actual increase in the perfusion flow after drink consumption. Each of those contrast images was then entered into a third-level paired-sample $t$ test, which compared the drink interventions. The resulting $Z$ (Gaussianised T/F) statistic image was then cluster thresholded with initial clusters determined using a voxel-wise uncorrected height threshold of $Z>2.3$ followed by a cluster significance threshold of $P<0.05$ (corrected for multiple comparisons). Before analysis, normality checks were performed on all data, and outliers were removed.

\section{Results}

\section{Arterial spin labelling cerebral blood flow}

Fig. 1 shows significantly greater regional perfusion in the inferior frontal gyrus and middle frontal gyrus of the right hemisphere $2 \mathrm{~h}$ following consumption of the HF drink compared with the CT drink (988 voxels, coordinates: $(X=37.9$, $Y=31 \cdot 8, \quad Z=17 \cdot 8$ ), statistics threshold: $Z=3.69, \quad P<0.001)$. There were no significant differences in regional perfusion between the HF and CT drinks $5 \mathrm{~h}$ after consumption, and no significant differences in global perfusion were observed between the two conditions at either time point.

\section{Cognitive tests}

A significant drink $\times$ time interaction was observed for the DSST $\left(F_{1,23}=10 \cdot 76, P<0 \cdot 01\right)$. As shown in Fig. 2, post hoc $t$ tests revealed that consumption of the HF drink resulted in a significant improvement in DSST performance at $2 \mathrm{~h}$ relative to baseline $(t=3 \cdot 84, P<0 \cdot 01)$, whereas no significant improvement in performance was observed following the CT drink $(t=0.05, P=0.96)$. Baseline DSST performance did not

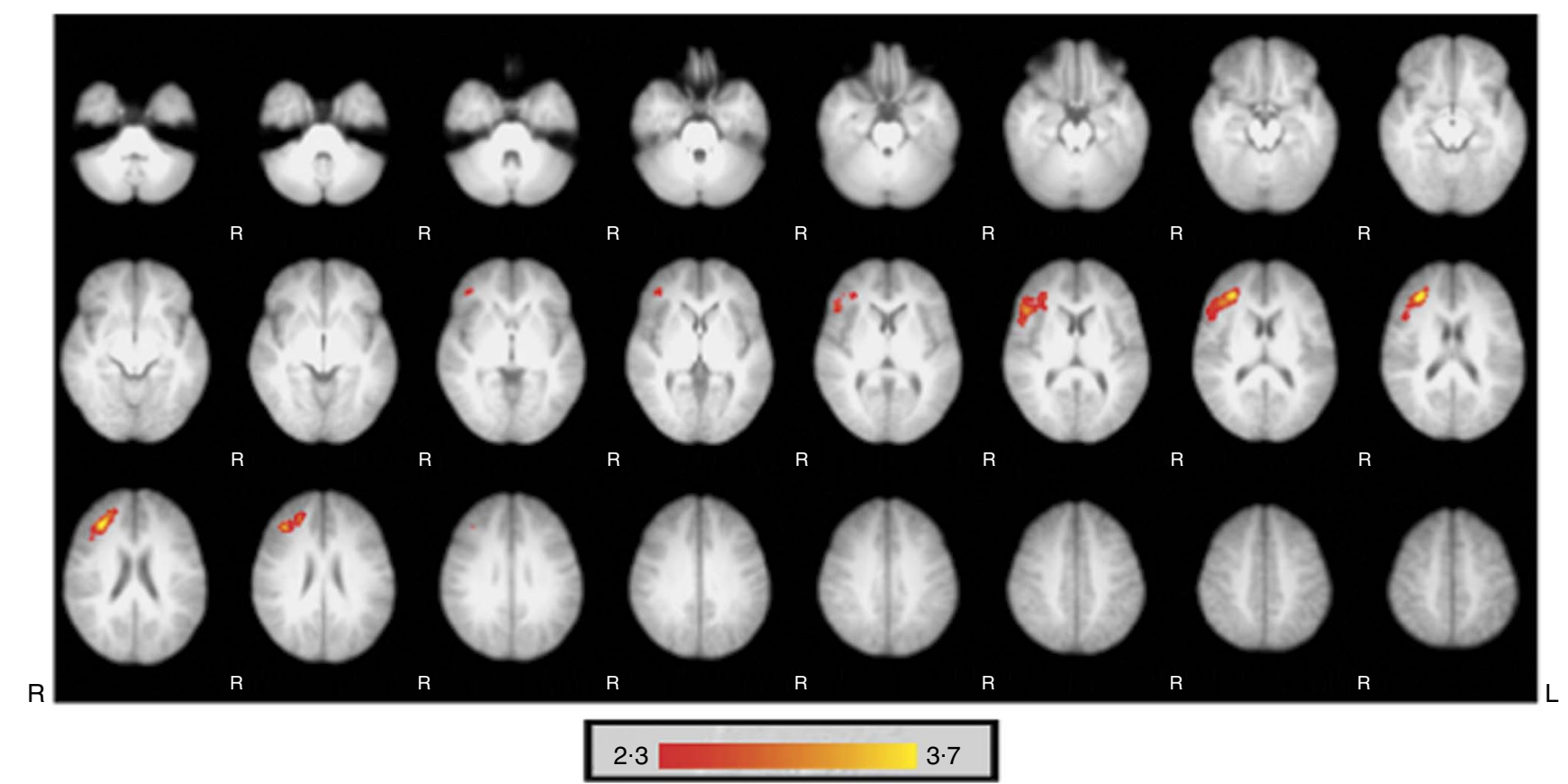

Fig. 1. Significantly greater regional perfusion occurred in the inferior frontal gyrus and the medial frontal gyrus of the right hemisphere $2 \mathrm{~h}$ following the high-flavanone drink compared with the control drink. Activations are superimposed on axial slices of the Montreal Neurological Institute template brain and represent perfusion flow in $\mathrm{ml} / 100 \mathrm{~g}$ tissue per min with $\square$ indicating greater perfusion. The images were initially thresholded at $Z>2.3$ to identify activation clusters, and then a (corrected) cluster significance threshold of $P<0.05$ was applied. 


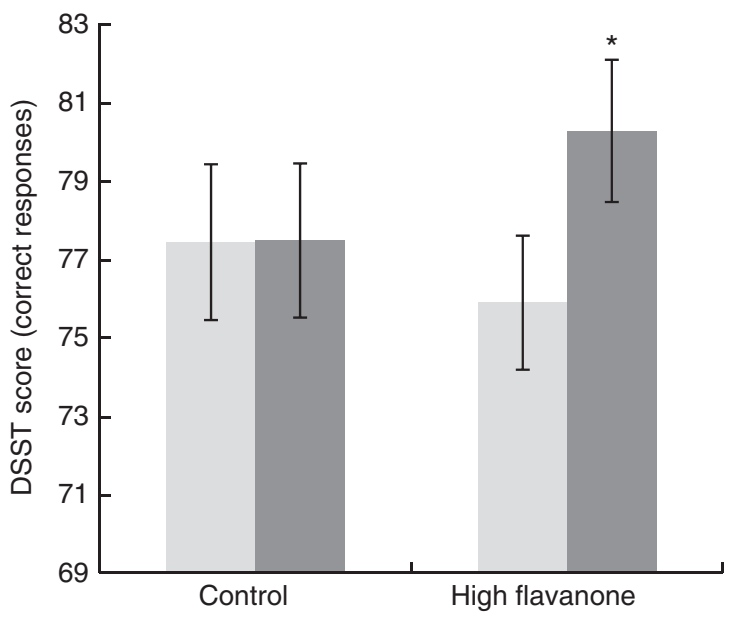

Fig. 2. Digit Symbol Substitution Test (DSST) mean correct responses and standard errors for the control and high flavanone drink at baseline and $2 \mathrm{~h}$ after consumption. Following a significant drink $\times$ time interaction $\left(F_{1,23}=10.76\right.$, $P<0.01)$, post hoc tests revealed that the number of correct responses on the DSST was significantly greater at $2 \mathrm{~h}(\square)$ relative to baseline $(\square)$ ( $t=3.84, \quad P<0.01$ ) following consumption of the flavanone-rich juice. ${ }^{*}$ Mean value was significantly different from that at baseline $(P<0.05$; post hoc test).

differ between the CT and HF drinks $(t=0.02, P=0.98)$. No significant interactions or main effects were observed for all other cognitive tests (see Table 2).

\section{Blood pressure}

The drink $\times$ time interactions were not significant for either diastolic $\left(F_{1,23}=1 \cdot 19, \quad P=0 \cdot 29\right)$ or systolic blood pressure $\left(F_{1,23}=0.5, P=0.49\right)$. However, main effects of time revealed that both systolic $\left(F_{1,23}=4.56, \quad P<0.05\right)$ and diastolic $\left(F_{1,23}=13.38, P<0.01\right)$ blood pressure significantly reduced at $2 \mathrm{~h}$ relative to baseline (see Table 2 ). To further explore the main effect of time, post hoc $t$ tests revealed that consumption of the HF drink significantly reduced diastolic blood pressure at 2 h compared with baseline $(t=3.43, P<0 \cdot 01)$, whereas this reduction did not reach significance following the CT drink $(t=2 \cdot 05, P>0 \cdot 05)$.

\section{Discussion}

Acute improvement in the measure of executive function (DSST) and increased $\mathrm{CBF}$ in the right frontal gyrus during conscious resting state were observed $2 \mathrm{~h}$ following consumption of $500 \mathrm{ml}$ of flavanone-rich citrus juice relative to a zero-flavonoid, vitamin C-matched, equienergetic CT drink. These data indicate that 70.5-mg flavonoids (specifically $42 \cdot 15-\mathrm{mg}$ hesperidin, 17.25-mg naringin, $6 \cdot 75-\mathrm{mg}$ narirutin, $4 \cdot 3-\mathrm{mg}$ caffeic acid) can increase CBF in healthy, young adults. However, these data do not provide evidence for a direct association between increased $\mathrm{CBF}$ and behavioural benefits. First, cognitive testing and CBF were not assessed simultaneously, and, moreover, no effects were observed for the majority of cognitive outcomes.
Table 2. Cognitive test and blood pressure (BP) data at baseline and $2 \mathrm{~h}$ after consumption of the control and high-flavanone drinks (Mean values and standard deviations)

\begin{tabular}{|c|c|c|c|c|c|}
\hline & \multicolumn{2}{|c|}{ Control drink } & \multicolumn{2}{|c|}{ High flavanone } & \multirow{2}{*}{$\begin{array}{c}\text { Drink } \times \text { time } \\
\text { interaction }(P)\end{array}$} \\
\hline & Mean & SD & Mean & $\mathrm{SD}$ & \\
\hline \multicolumn{6}{|l|}{ DSST } \\
\hline Baseline & $77 \cdot 4$ & $9 \cdot 7$ & 75.9 & 8.4 & \multirow{2}{*}{$0.003^{\star *}$} \\
\hline $2 \mathrm{~h}$ & 77.5 & 9.6 & $80 \cdot 3$ & 8.9 & \\
\hline \multicolumn{6}{|c|}{ FVT Landolt C } \\
\hline Baseline & 0.41 & 0.03 & 0.4 & 0.02 & \multirow[t]{2}{*}{0.19} \\
\hline $2 \mathrm{~h}$ & 0.42 & 0.04 & 0.4 & 0.02 & \\
\hline \multicolumn{6}{|l|}{ FVT Vernier } \\
\hline Baseline & $21 \cdot 2$ & $23 \cdot 3$ & $19 \cdot 9$ & $20 \cdot 1$ & \multirow[t]{2}{*}{0.65} \\
\hline $2 \mathrm{~h}$ & $19 \cdot 6$ & $16 \cdot 8$ & $21 \cdot 3$ & 14.7 & \\
\hline \multicolumn{6}{|l|}{ Go-NoGo } \\
\hline Baseline & 315 & 55 & 310 & 60 & \multirow[t]{2}{*}{0.86} \\
\hline $2 \mathrm{~h}$ & 308 & 62 & 305 & 57 & \\
\hline \multicolumn{6}{|c|}{ Letter Memory } \\
\hline Baseline & 77 & $16 \cdot 7$ & 74.6 & 18.4 & \multirow[t]{2}{*}{0.89} \\
\hline $2 \mathrm{~h}$ & $77 \cdot 1$ & 12 & $74 \cdot 1$ & $16 \cdot 3$ & \\
\hline \multicolumn{6}{|c|}{ Logical Memory Imm. $\dagger$} \\
\hline Baseline & 17.5 & 3.6 & $18 \cdot 3$ & $3 \cdot 3$ & \multirow[t]{2}{*}{0.97} \\
\hline $2 \mathrm{~h}$ & $15 \cdot 4$ & 3 & $16 \cdot 1$ & 3.6 & \\
\hline \multicolumn{6}{|c|}{ Logical Memory Del. $†$} \\
\hline Baseline & $16 \cdot 1$ & 3.6 & $15 \cdot 8$ & 3.9 & \multirow[t]{2}{*}{0.48} \\
\hline $2 \mathrm{~h}$ & $14 \cdot 1$ & 3.8 & $14 \cdot 6$ & 3.3 & \\
\hline \multicolumn{6}{|c|}{ Sequence Learning } \\
\hline Baseline & 97.8 & 1.5 & 98 & 1.6 & \multirow{2}{*}{0.52} \\
\hline $2 \mathrm{~h}$ & $96 \cdot 9$ & $2 \cdot 1$ & 97 & 2 & \\
\hline \multicolumn{6}{|c|}{ Spatial Memory } \\
\hline Baseline & $27 \cdot 3$ & $15 \cdot 8$ & 28.2 & 18 & \multirow[t]{2}{*}{0.68} \\
\hline $2 \mathrm{~h}$ & $28 \cdot 2$ & $15 \cdot 4$ & 30 & $20 \cdot 6$ & \\
\hline \multicolumn{6}{|l|}{ Stroop } \\
\hline Baseline & 654 & 74 & 647 & 71 & \multirow[t]{2}{*}{0.71} \\
\hline $2 \mathrm{~h}$ & 626 & 84 & 623 & 67 & \\
\hline \multicolumn{6}{|c|}{ Word Recall Imm. } \\
\hline Baseline & 7.3 & 3.2 & $7 \cdot 3$ & 3.5 & 0.11 \\
\hline $2 \mathrm{~h}$ & 7 & $2 \cdot 7$ & $5 \cdot 7$ & 2.5 & \\
\hline Word Recall & el.ł & & & & \\
\hline Baseline & $5 \cdot 2$ & $2 \cdot 9$ & $5 \cdot 2$ & $3 \cdot 2$ & 0.15 \\
\hline $2 \mathrm{~h}$ & 4.5 & 2.5 & $3 \cdot 2$ & $2 \cdot 3$ & \\
\hline Diastolic BP & $\mathrm{nmHg}$ ) & & & & \\
\hline Baseline & 72 & 8.4 & $71 \cdot 7$ & 7.5 & 0.49 \\
\hline $2 \mathrm{~h}$ & $69 \cdot 7$ & $7 \cdot 8$ & 68.4 & 7.5 & \\
\hline Systolic BP & $\mathrm{imHg}$ ) & & & & \\
\hline Baseline & 115.9 & $12 \cdot 4$ & 116.5 & $12 \cdot 4$ & 0.29 \\
\hline $2 \mathrm{~h}$ & $115 \cdot 3$ & $12 \cdot 3$ & $113 \cdot 8$ & $12 \cdot 1$ & \\
\hline
\end{tabular}

DSST, Digit Symbol Substitution Test, correct responses; FVT Landolt C, Freiburg Vision Test Landolt C, a higher score indicates better vision; FVT Vernier, Freiberg Vision Test Vernier Threshold, a higher score indicates better vision; Go-NoGo, GoNoGo reaction time (ms); Letter Memory, Letter Memory Accuracy; Sequence Learning, Sequence Learning correct responses; Spatial Memory, Spatial Delayed Recall Test distance from target $(\mathrm{mm})$; Stroop, Computerised Stroop reaction time $(\mathrm{ms})$. ${ }^{\star *} P<0.01$.

$\dagger$ Logical Memory units recalled.

¥ Word Recall number of words recalled.

This is the first data set to show region-specific increases in human CBF following a flavanone dose. The frontal gyrus has been identified within a network of brain areas that are active during conscious resting state ${ }^{(23)}$, which may explain the observed region-specific increased perfusion. The inferior frontal gyrus has typically been implicated in tasks that require inhibition, planning, decision making and other aspects of executive function ${ }^{(24)}$, such as the DSST, for which improvements were observed in this study following the flavanone-rich juice. However, the mechanisms underpinning the right hemispheric lateralisation are unclear. 
These data provide evidence that flavonoid sub-classes other than cocoa flavanols can also have acute effects on CBF within the immediate postprandial period. Increased global CBF across the grey matter was observed $2 \mathrm{~h}$ after consumption of a 560-mg flavanol drink relative to a CT drink $^{(12)}$; however, regional blood flow was not assessed, most likely due to the small sample size of healthy young adults $(n 4)$. The same authors also reported that a smaller flavanol dose $(172 \mathrm{mg})$ was associated with increased region-specific BOLD signal intensity (including medial and lateral prefrontal cortex, parietal cortex, anterior cingulate cortex and the cerebellum) $1.5 \mathrm{~h}$ after consumption in sixteen health, young adults, although the cocoa drink was consumed for 5 consecutive days before the fMRI scan. Direct comparisons between the regions of interest reported by Francis et al. ${ }^{(12)}$ and the present study are restricted by differences in scanning methods (BOLD or ASL), the flavonoid sub-class and dose (172-mg cocoa flavanols or $70 \cdot 5-\mathrm{mg}$ fruit flavanones), duration of consumption ( $5 \mathrm{~d}$ or a single acute dose) and behavioural instructions during imaging; the present study examined conscious resting state, whereas Francis et $a l .{ }^{(12)}$ examined neural activity during an executive function task. In addition, a limitation of the present study was the absence of double blinding during data collection, which could have introduced experimenter biases. Critically, however, data analysis was performed blinded by an independent researcher. Further investigations of the acute effects of flavonoid consumption on regional $\mathrm{CBF}$ are required in order to identify whether specific regions appear to particularly react to flavonoid ingestion in the postprandial period. For example, increased perfusion in the anterior cingulate cortex and central opercular cortex was recently observed $2 \mathrm{~h}$ after consumption of 494-mg cocoa flavanols ${ }^{(25)}$; however, behavioural tasks were not assessed. Studies of neural activation following chronic daily consumption of fruit-based flavonoids ${ }^{(9)}$ and flavanol-rich cocoa flavonoids ${ }^{(13,14)}$ indicate that areas of the brain implicated in memory function such as the hippocampus, specifically the dentate gyrus, are especially sensitive.

The mechanisms by which flavonoids acutely induce vasodilation and enhance $\mathrm{CBF}$ are thought to be via increased nitric oxide synthesis in the endothelium. Nitric oxide synthesis is a key regulator of angiogenesis and the dilation of cells, and is also synthesised by neurons in response to neuronal activation $^{(26)}$. As such, nitric oxide is thought to be crucial for the coupling between increased blood supply and neuronal activity $^{(27)}$. Flavonoid ingestion in humans is known to enhance circulating nitric oxide species ${ }^{(28)}$ in association with beneficial vascular outcomes such as increased flow-mediated dilation and augmented microcirculation ${ }^{(11)}$. Therefore, it is plausible that flavonoid-induced increases in the bioavailability of nitric oxide in the brain may lead to increased blood vessel and neuronal efficiency and, subsequently, improvements in cognitive function. These vascular mechanisms are tentatively supported by the observed reduction in systolic blood pressure following the flavanone-rich juice in the present study; however, it should be noted that this was a subtle reduction $(3 \mathrm{mmHg})$. Having said that, a large reduction in blood pressure would not be anticipated in this sample of healthy young adults. Studies in adults with the metabolic syndrome have shown that
$550 \mathrm{mg}$ daily supplementation of the flavanone hesperidin for 3 weeks can lead to increased flow-mediated dilation and endothelial nitric oxide synthesis ${ }^{(29)}$. This is pertinent to the present findings, given that hesperidin was the predominant flavanone within the flavanone-rich citrus juice.

Further studies are required to directly examine the relationship between flavonoid consumption, nitric oxide activity, CBF and cognitive function. Interestingly, increased nitric oxide status in the plasma has been observed $2 \mathrm{~h}$ after consumption of flavonoid-rich apples; however, no effects were observed for cognitive function ${ }^{(30)}$. Kean et al $^{(10)}$ reported global cognitive improvements in cognition among healthy older adults following daily chronic consumption of flavanone-rich orange juice $(305 \mathrm{mg} / \mathrm{d})$ over 8 weeks; however, nitric oxide status was not examined. This sample of highly educated, healthy, young adults is likely performing close to optimal functioning, and therefore there is greater potential for acutely enhancing cognition in older adults who may be experiencing naturally occurring ageing associated cognitive decline. This may explain why effects were not observed for the majority of cognitive outcomes in the present study, particularly given the relatively small flavanone dose $(70.5 \mathrm{mg})$. Previously, positive behavioural effects in healthy, young adults have only been observed following high doses of cocoa flavanols - for example, $573 \mathrm{mg}^{(31)}$ and $550 \mathrm{mg} /$ $994 \mathrm{~g}^{(32)}$. In addition, it has been argued that flavonoid interventions are more likely to benefit cognition during tasks of high demand ${ }^{(32)}$; therefore, it is possible that the current cognitive battery was not suitably challenging. However, there was no evidence of ceiling effects.

It can be hypothesised that stronger behavioural effects may occur at a later time point, given that plasma flavanone metabolites following orange juice consumption have been observed to peak at $6 \mathrm{~h}^{(33,34)}$. Indeed, it is a limitation of the present study that cognitive function was exclusively assessed $2 \mathrm{~h}$ after consumption (in addition to baseline). Recently, benefits for global cognitive function and subjective alertness were observed 2 and $6 \mathrm{~h}$ after consumption of a flavanone-rich $(272 \mathrm{mg}) 100 \%$ orange juice in healthy, young adults, with the effects being more pronounced (relative to the CT drink) at $6 \mathrm{~h}^{(35)}$. Having said that, at present, increased CBF was observed at $2 \mathrm{~h}$ but not $5 \mathrm{~h}$, possibly indicating that the time course by which the flavonoids in orange and grapefruit juice exert their physiological effects may differ relative to $100 \%$ orange juice, although the mechanism for this is unclear. Future acute interventions of flavonoid consumption should examine plasma flavonoid metabolites concomitantly with cognitive outcomes to investigate whether peak metabolite concentrations coincide with the hypothesised behavioural effects. Flavanone metabolites are certainly of interest given that they are known to cross the blood-brain barrier ${ }^{(36)}$. Future studies should carefully consider the time span over which circulating flavonoid metabolites may impact cognitive outcomes. Anthocyanin metabolites have been observed in urine up to $5 \mathrm{~d}$ following acute ingestion of blueberries ${ }^{(36)}$. This has implications for the current findings; the 24-h dietary restriction may not have been sufficient to account for potential confounding effects of habitual flavonoid intake, although it is unclear whether the associated levels of circulating metabolites can acutely affect cognition. 
In conclusion, 500-ml citrus juice containing 70.5-mg flavonoids was associated with increased regional perfusion in the right frontal gyrus in young, healthy adults $2 \mathrm{~h}$ following consumption of the flavanone-rich juice in conscious resting state relative to the zero-flavonoid, equienergetic, vitamin C-matched control. These data demonstrate that fruit-based flavonoids can acutely enhance CBF in healthy adults. Behavioural improvements on a battery of cognitive tests following the flavonoid-rich juice were only observed for one measure of executive function (DSST) in a separate cohort of young adults. Therefore, the present data do not show a clear association between increased CBF and behavioural benefits. Further studies should simultaneously examine cognitive performance and respective functional brain activation, regional $\mathrm{CBF}$ and concentrations of circulating nitric oxide species following consumption of flavonoid-rich juices to further our understanding of the underlying mechanisms.

\section{Acknowledgements}

This research was funded by PepsiCo Inc.

J. M. F., L. T. B. and J. P. E. S. designed the study and reviewed and edited the manuscript. D. J. L., D. P. and A. L. M. analysed the data and prepared the manuscript. A. L. M., S. B.-B. and D. P. conducted the study.

The authors declare that there are no conflicts of interest.

\section{References}

1. Spencer JPE (2010) Beyond antioxidants: the cellular and molecular interactions of flavonoids and how these underpin their actions on the brain. Proc Nutr Soc 69, 244-260.

2. Menze ET, Tadros MG, Abdel-Tawed AM, et al. (2012) Potential neuroprotective effects of hesperidin on 3-nitropropuinic acidinduced neurotoxicity in rats. Neurotoxicology 33, 1265-1275.

3. Rendeiro C, Vauzour D, Rattray M, et al. (2013) Dietary levels of pure flavonoids improve spatial memory performance and increase hippocampal brain derived neurotrophic factor. PLOS ONE 8, e63535.

4. Laske C, Stellos K, Hoffmann N, et al. (2011) Higher BDNF serum levels predict slower cognitive decline in Alzheimer's disease patients. Int J Neuropsychopharmacol 14, 399-404.

5. Butchart C, Kyle J, McNeill G, et al. (2011) Flavonoid intake in relation to cognitive function in later life in the Lothian Birth Cohort 1936. Br J Nutr 106, 141-148.

6. Lamport DJ, Dye L, Wightman JD, et al. (2012) The effects of flavonoid and other polyphenol consumption on cognitive performance: a systematic research review of human experimental and epidemiological studies. Nutr Aging 1, 5-25.

7. Lamport DJ, Hoyle E, Lawton CL, et al. (2011) Evidence for a second meal cognitive effect: glycaemic responses to high and low glycaemic index meals are associated with cognition the following morning. Nutr Neurosci 14, 66-71.

8. Krikorian R, Nash TA, Shidler MD, et al. (2010) Concord grape juice supplementation improves memory function in older adults with mild cognitive impairment. Br J Nutr 103, 730-734.

9. Krikorian R, Boespflug EL, Fleck DE, et al. (2012) Concord grape juice supplementation and neurocognitive function in human aging. J Agric Food Chem 60, 5736-5742.

10. Kean RJ, Lamport DJ, Dodd GF, et al. (2015) Chronic consumption of flavanone-rich orange juice is associated with cognitive benefits: an 8-week randomised double-blind placebo-controlled trial in healthy older adults. Am J Clin Nutr 101, 506-514.

11. Schroeter H, Heiss C, Balzer J, et al. (2006) (-)-Epicatechin mediates beneficial effects of flavanol-rich cocoa on vascular function in humans. Proc Natl Acad Sci U S A 103, 1024-1029.

12. Francis S, Head K, Morris PG, et al. (2006) The effect of flavanol-rich cocoa on the fMRI response to a cognitive task in healthy young people. J Cardiovasc Pharmacol $\mathbf{4 7}$, S215-S220.

13. Brickman AM, Khan US, Provenzano FA, et al. (2014) Enhancing dentate gyrus function with dietary flavanols improves cognition in older adults. Nat NeuroSci 17, 1798-1803.

14. Camfield DA, Scholey A, Pipingas A, et al. (2012) Steady state visually evoked potential (SSVEP) topography changes associated with cocoa flavanol consumption. Physiol Behav 105, 948-957.

15. Krikorian R, Shidler MD, Nash TA, et al. (2010) Blueberry supplementation improves memory in older adults. J Agric Food Chem 58, 3996-4000.

16. Lamport DJ, Saunders C, Butler LT, et al. (2014) Fruits, vegetables, 100\% juices and cognitive function. Nutr Rev 72, 774-789.

17. Bach M (1996) The Freiburg Visual Acuity Test - automatic measurement of visual acuity. Optom Vis Sci 73, 49-53.

18. Stadler MA (1995) Role of attention in implicit learning. J Exp Psychol Learn 21, 674-685.

19. Wechsler D (1981) The psychometric tradition - developing the Wechsler adult intelligence scale. Contemp Educ Psychol 6, 82-85.

20. Stroop JR (1935) Studies of interference in serial verbal reactions. J Exp Psychol 18, 643-662.

21. Morris N \& Jones DM (1990) Memory updating in working memory: the role of the central executive. Br J Psychol 81, 111-121.

22. Garavan H, Ross TJ \& Stein EA (1999) Right hemispheric dominance of inhibitory control: an event-related functional MRI study. Proc Natl Acad Sci U S A 96, 8301-8306.

23. Binder JR, Frost JA, Hammeke TA, et al. (1999) Conceptual processing during the conscious resting state: a functional MRI study. J Cogn Neurosci 11, 80-93.

24. Aron AR, Robbins TW \& Poldrack RA (2004) Inhibition and the right inferior frontal cortex. Trends Cogn Sci 8, 170-177.

25. Lamport DJ, Pal D, Moutsiana C, et al. (2015) The effect of flavanol rich cocoa on cerebral perfusion in healthy older adults during conscious resting state: a placebo controlled crossover acute trial. Psychopharmacology (Berl) 232, $3227-3234$

26. Berdt DS, Hwang PM \& Snyder SH (1990) Localization of nitric oxide synthesis indicating a neural role for nitric oxide. Nature 347, 768-770

27. Toda N, Ayajiki K \& Okamura T (2009) Cerebral blood flow regulation by nitric oxide in neurological disorders. Can J Physiol Pharmacol 87, 581-594.

28. Loke WM, Hodgson JM, Proudfoot JM, et al. (2008) Pure dietary flavonoids quercetin and (-)-epicatechin augment nitric oxide products and reduce endothelin- 1 acutely in healthy men. Am J Clin Nutr 88, 1018-1025.

29. Rizza S, Muniyappa R, Lantorno M, et al. (2011) Citrus polyphenol hesperidin stimulates production of nitric oxide in endothelial cells while improving endothelial function and reducing inflammatory markers in patients with metabolic syndrome. J Clin Endocrinol Metab 96, E782-E792.

30. Bondonno CP, Downey LA, Croft KD, et al. (2014) The acute effect of flavonoid-rich apples and nitrate rich spinach on cognitive performance and mood in healthy men and women. Food Funct 5, 849-858. 
31. Field DT, Williams CM \& Butler LT (2011) Consumption of cocoa flavanols results in an acute improvement in visual and cognitive functions. Physiol Behav 103, 255-260.

32. Scholey A, French SJ, Morris PJ, et al. (2010) Consumption of cocoa flavanols results in acute improvements in mood and cognitive performance during sustained mental effort. J Psychopharmacol 24, 1505-1514.

33. Manach C, Scalbert A \& Morand C (2004) Polyphenols: food sources and bioavailability. Am J Clin Nutr 79, 27-47.
34. Mullen W, Archeveque MA, Edwards CA, et al. (2008) Bioavailability and metabolism of orange juice flavanones in humans: impact of a full-fat yogurt. J Agric Food Chem 56, 11157-11164.

35. Alharbi MH, Lamport DJ, Dodd G, et al. (2016) Flavonoid rich orange juice is associated with acute improvements in cognitive function in healthy middle aged males. Eur J Nutr 55, 2021-2029.

36. Youdim KA, Dobbie MS, Kuhnle G, et al. (2003) Interaction between flavonoids and the blood-brain barrier: in vitro studies. J Neurochem 85, 180-192. 